\title{
Computational study of flow around a NACA 0012 by using Roe FVM scheme and davis-yee TVD scheme
}

\author{
Fatimah Yusop ${ }^{1}$, Zamri Omar ${ }^{2}$, Bambang Basuno ${ }^{3}$, Nik Normunira Mat Hassan ${ }^{4}$ \\ ${ }^{1,4}$ Faculty of Engineering Technology, Universiti Tun Hussein Onn Malaysia, Malaysia \\ ${ }^{2,3}$ Faculty of Mechanical and Manufacturing Engineering, Universiti Tun Hussein Onn Malaysia, Malaysia
}

\begin{tabular}{l} 
Article Info \\
\hline Article history: \\
Received Jan 12, 2019 \\
Revised Mar 10, 2019 \\
Accepted Apr 15, 2019 \\
\hline
\end{tabular}

Keywords:

Computational fluid dynamics upwind scheme

\begin{abstract}
Currently CFD had been considered as an important tool for solving engineering problems. The application of CFD had been used intensively in aircraft industries in design a new aircraft or in the effort of improvement on the exiting aircraft. In term of CFD computer code, the CFD code differs with any others may due to the difference in the numerical scheme have been used. Therefore, the present work presents the comparison result between two developed computer codes with ANSYS-FLUENT software to the case of transonic steady flow past through airfoil NACA 0012. The first computer code used a finite difference method with numerical scheme according to Davis-Yee TVD scheme. Meanwhile, the second computer code used a Roe's cell centre finite volume scheme. The flow analysis is carried out at two Mach number, M (0.65 \& 0.8). Each Mach number applied to two different angles of attacks $\left(0^{\circ} \& 5^{\circ}\right)$. The flow domain discretized by use of C-topology with $193 \times 63$ grid points. The comparison in term of the pressure coefficient, along the airfoil surface are presented. From the result, indicated that developed computer code is able to capture the presence of shock wave in the flow field.
\end{abstract}

Copyright $(2019$ Institute of Advanced Engineering and Science. All rights reserved.

Corresponding Author:

Fatimah Yusop,

Faculty of Engineering Technology,

Universiti Tun Hussein Onn Malaysia,

Pagoh Higher Education Hub, 84600 Pagoh, Muar, Johor.

Email: fatimahy@uthm.edu.my

\section{INTRODUCTION}

Computational fluid dynamics (CFD) is very widespread use in engineering activities which involves in solving fluid flow problems. The governing equations of fluid motion called the Navier-Stokes equations are well established nearly one and half centuries ago [1]. Tinoco estimated that direct solution to the Navier-Stokes in line with progress in the development of computing power and the algorithm in solving the non-linear partial differential equation can be made in the next 60 years from now [2]. However for particular flow problem especially for the case of high Reynolds number flow past through a streamline body at relatively low angle of attack, the governing equation for such kind flow can be well presented by the Euler equations $[3,4]$.

For the case of transonic steady flow problems, the Euler equation can behave as Elliptic partial differential equation or hyper-bolic partial differential equation depending on the local Mach number [5]. In the presence such mixed types behavior, makes the Euler equation becomes difficulties to be solved. Such difficulty can be removed by treating the governing equa-tion of fluid motion in the form time dependent equation. In this respect the Euler equation behaves as hyperbolic equation with respect to time over the whole flow domain. There are various methods had been developed for solving the Euler equation in corresponding to the transonic steady flow problem. 
The present work deals with the flow problem related to stream-line body of a relatively low angle of attack in which the viscous effect can be neglected. In solving this type of flow, the two computer codes are developed and designed. The first computer code are developed based on finite difference method according to Davis-Yee TVD scheme $[6,7]$, while the second computer code uses a finite volume approach by adopting the scheme introduced by Roe $[8,9]$.

\section{RESEARCH METHOD}

The methodology of this study is based on CFD code development. Basically, CFD code involves three process, called pre-processing, solver and post processing with sequence as depicted in Figure 1. Pre-processing involves the way the flow domain of the flow problem under investigation is discretized. Solver related in manner of the governing equation of fluid motion is solved. The post processing related in the result of flow properties presented [10]. In text of solver, which involves how to solve the governing equation of fluid motion, it can use a numerical scheme developed according to finite difference method (FDM) or finite volume method (FVM). The present work involves the solver developed based on FDM as well as FVM. Meanwhile, Figure 2 shows the general flow in solver code. The processes start with defining the simulation parameter until generates the final result.

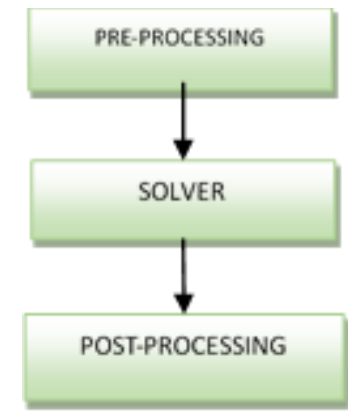

Figure 1. General flow of CFD code development

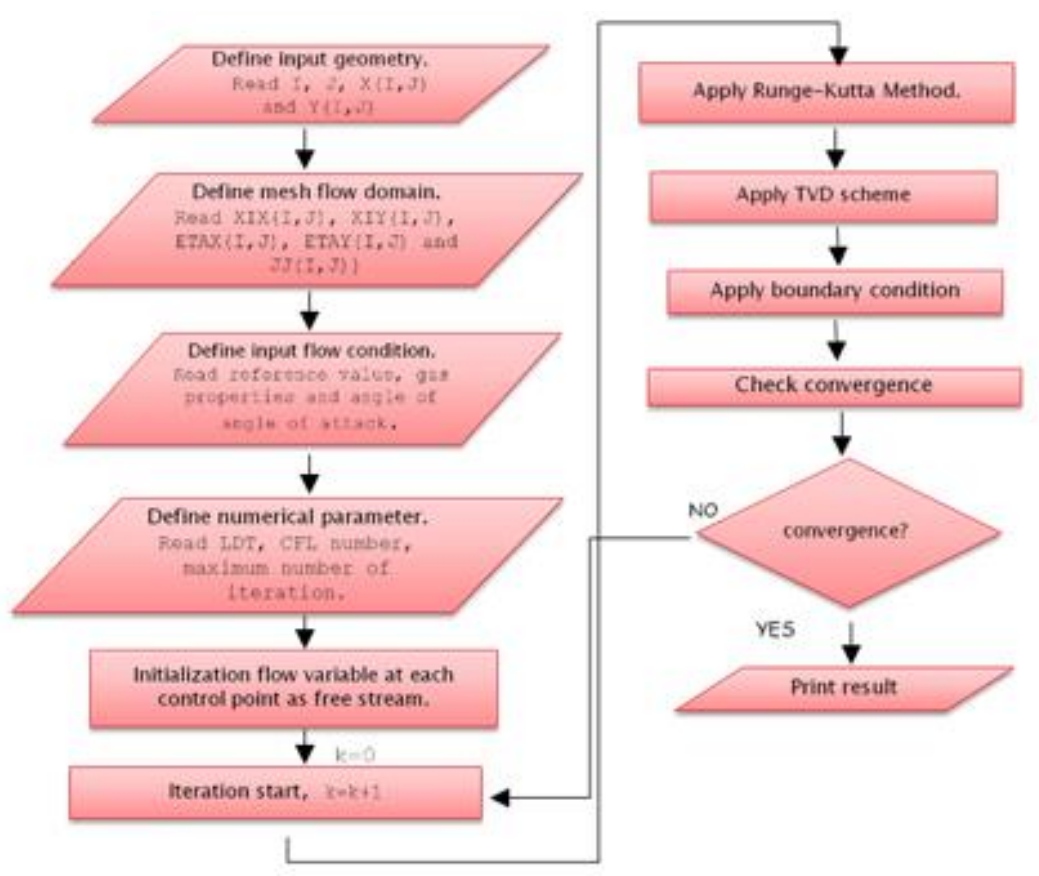

Figure 2. Flow of CFD solver 


\subsection{Structured Grid Generation}

This study was implemented C-topology in defining the flow domain past through airfoil and have been used by the Elliptic grid generator and algebraic generator as its grid initialization with spatial discretization in $\xi$ and $\eta$ direction with 193 points and 63 points respectively gives the result as shown in Figure 3.

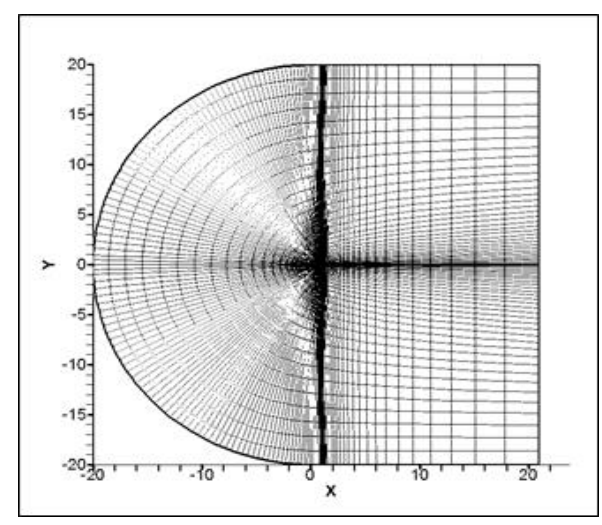

Figure 3. C-grid of NACA 0012

\subsection{Governing Equation}

For the compressible flow past through a streamline body at relatively low angle of attack and high Reynold number can be approximated as one solves an inviscid flow problem. Hence such flow phenomena can be well presented by the Euler equations. The unsteady, two dimensional compressible inviscid flows make the Euler equations in differential and in conservative form and vector notation can be written as $[11,12]$ :

$$
\frac{\partial Q}{\partial t}+\frac{\partial E}{\partial x}+\frac{\partial F}{\partial y}=0
$$

In above equation $\mathrm{Q}$ is a state vector of dependent variables while $\mathrm{E}$ and $\mathrm{F}$ are the flux vector in $\mathrm{x}$ and y direction respectively. These three vectors are defined as:

$$
Q=\left[\begin{array}{c}
\rho \\
\rho u \\
\rho v \\
\rho e_{t}
\end{array}\right], E=\left[\begin{array}{c}
\rho u \\
\rho u^{2} \\
\rho u v \\
\rho u\left(e_{t}+\frac{p}{\rho}\right)
\end{array}\right], F=\left[\begin{array}{c}
\rho v \\
\rho v u \\
\rho v^{2}+p \\
\rho v\left(e_{t}+\frac{p}{\rho}\right)
\end{array}\right]
$$

and

$$
e_{t}=e+\frac{u^{2}+v^{2}}{2}
$$

The variables are appearing in (2) have definition as commonly used in fluid dynamics study. The variable $\rho$ represents the air density, $u$ and $v$ are the component velocities in $x$ and $y$ direction. et is the total internal energy per unit of mass and $\mathrm{p}$ is the pressure. Considering (2), it is clearly indicating that (1) consist four equations with five dependent variables. One additional relationship is required to make (1) as solvable equation. By considering the flow behaves as a perfect gas, the additional relationship can be defined from the relationship between the internal energy per unit of mass e and other flow variables as:

$$
e=\frac{p}{\rho(\gamma-1)}
$$

In integral form, the Euler equation $\mathrm{n}$ can be presented as [13]: 


$$
\frac{\partial}{\partial t} \int_{0}^{\Omega} Q d \Omega+\int_{0}^{S}\left(E n_{x}+F n_{y}\right) d S=0
$$

In above equation, $\Omega$ is a cell volume, $\mathrm{nx}$ and ny are the component of the normal unity vector on the cell faces. $\mathrm{S}$ is the cell surface area. While variable Q, E and F have the same definition as in the Euler equation in its differential form.

\subsection{TVD Scheme}

TVD scheme is a high-resolution scheme that was developed to solve the problem related to nonlinear hyperbolic conservation laws. The term TVD was introduced by Harten in 1983 [14]. Overall, TVD scheme is not as specific as a scheme but more as properties. Therefore, TVD scheme contains a variety of scheme. That means, TVD scheme can be summarized to have the following physical characteristics [15-17].

a) Entropy condition - A decrease of entropy associated with expansion shocks must not be admitted.

b) Monotonic condition - This condition must be enforced to prevent oscillatory behavior in the numerical scheme.

c) Total Variation Diminishing (TVD) - The total variation of any physically admissible solution must not be allowed to increase time. Basically, can be defined as follow:

$$
T V=\int\left|\frac{\partial u}{\partial x}\right| d x
$$

Through above criteria, it can develop a manner on how to discretize the governing equation of fluid motion. Among them are TVD scheme according to [18-20]. The CFD code which developed based on TVD criteria had been widely used as CFD code for aircraft aerodynamics analysis [21]. In term of fluid flow analysis, TVD scheme had been used extensively especially for internal flow problem such in [22, 23] and [24]. Therefore, this study developed the TVD scheme based on Davis-Yee scheme for solve external flow that able to solve the aerodynamics analysis as shown in (6).

$$
\bar{Q}_{i, j}^{n+1}=\bar{Q}_{i, j}^{n}-\frac{1}{2} \frac{\Delta \tau}{\Delta \xi}\left[\left(X_{A}\right)_{i+\frac{1}{2}, j}^{n}\left(\Phi_{\xi}\right)_{i+\frac{1}{2}, j}^{n}-\left(X_{A}\right)_{i-\frac{1}{2}, j}^{n}\left(\Phi_{\xi}\right)_{i-\frac{1}{2} j}^{n}\right] \frac{1}{2} \frac{\Delta \tau}{\Delta \eta}\left[\left(X_{B}\right)_{i, j+\frac{1}{2}}^{n}\left(\Phi_{\eta}\right)_{i, j+\frac{1}{2}}^{n}-\left(X_{B}\right)_{i, j-\frac{1}{2}}^{n}\left(\Phi_{\eta}\right)_{i, j-\frac{1}{2}}^{n}\right]
$$

In above equation $\Phi$ is a flux limiter vector and $\mathrm{G}$ is limiter function.

\subsection{ROE Scheme}

The second code is developed by ROE scheme. This scheme is based on a characteristic decomposition of the flux differences [25]. The Roe scheme used flux formula at the interface of a control volume that is equal to the average fluxes of left and right states minus a differencing term which splits the difference of the fluxes on both sides of the control volume. The basic equation is expressed as below [8].

$$
\left(\vec{F}_{c}\right)_{R}-\left(\vec{F}_{c}\right)_{L}=\left(\bar{A}_{R O E}\right)_{I+1 / 2}\left(\vec{Q}_{R}-\vec{Q}_{L}\right)
$$

where ARoe is Roe matrix and $\mathrm{L}$ and $\mathrm{R}$ the left and right state respectively.

\section{RESULTS AND DISCUSSION}

The simulation was conducted in four different flow conditions with varying the Mach number $(0.8$ and 0.65$)$ and angle of attack $\left(0^{\circ}\right.$ and $\left.5^{\circ}\right)$. Comparison results are presented between the two developed codes (ROE FVM and TVD FDM) and with commercial code ANSYS-FLUENT.

\subsection{Air Flow on NACA 0012 Airfoil for $M=0.65$ and $\alpha=0^{\circ}$}

The comparison result in terms of pressure coefficient distribution along the airfoil surface is as shown in Figure 4. Both developed computer codes are in good agreement with each other compared to the ANSYS-FLUENT software results discrepancies appearing at $\mathrm{x}<0.4 \mathrm{c}$. The result also indicated the value for upper surface and lower surface at the same point due to the symmetrical airfoil with $0^{\circ}$ angle of attack. In terms of pressure coefficient value at stagnation point, developed code is nearly 1.0 compared to ANSYSFLUENT software obtained around 0.1. The comparison results in view of Mach number is shown in Figure 5. 


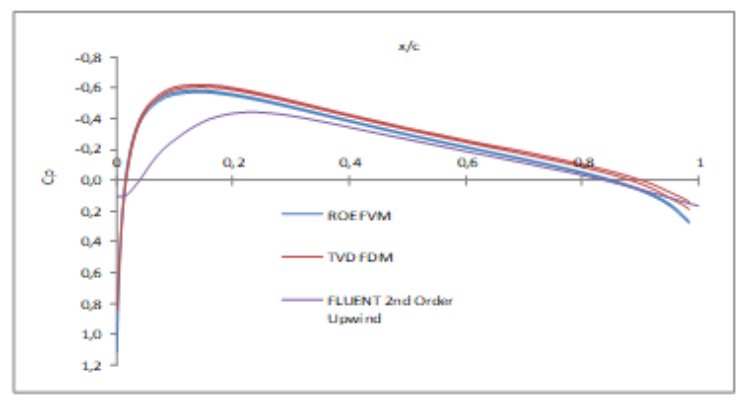

Figure 4. Pressure coefficient distribution at $\alpha=0^{\circ}$ and $M=0.65$

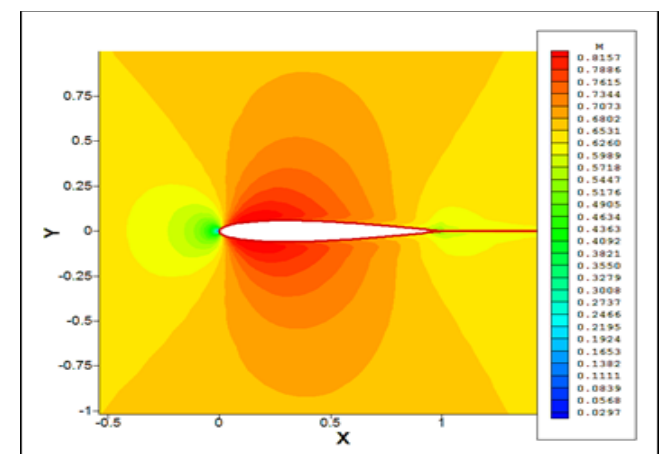

(a)

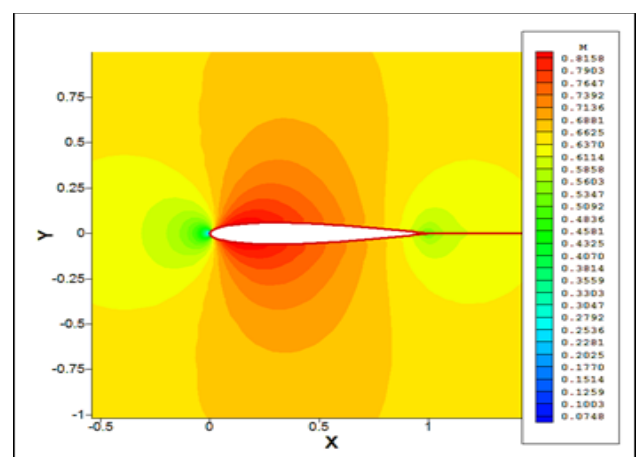

(b)

Figure 5. The Mach number contour near the NACA 0012, at $\alpha=0^{\circ}$ and $M=0.65$ through (a) TVD FDM and (b) Roe FVM

\subsection{Air Flow on NACA 0012 Airfoil for $M=0.65$ and $\alpha=5^{\circ}$}

A comparison result between the developed computer codes and ANSYS-FLUENT software for pressure coefficient along the airfoil surface at different angles of attack is shown in Figure 6. At this angle of attack, both developed computer codes (FDM Code and FVM Code) predict the presence of the shock wave, while ANSYS-FLUENT software does not indicate such phenomena. As a result, the pressure distribution distribution along the airfoil surface differs. However, the difference between the TVD FDM code and the Roe FVM code are as not much as the difference with ANSYS-FLUENT software. The difference of TVD FDM code and Roe FVM code are simply due to their difference in predicting the location of shock wave. However, both schemes are able to produce a sharp pressure gradient without wiggles or oscillation at their shock points. Table 1 shows the shock position on the upper surface of the airfoil for these three CFD codes. The flow pattern of Mach number distribution over the flow field for the TVD FDM and Roe FVM in Figure 7.

Table1. Comparison result shock position of NACA 0012 at $\alpha=5^{\circ}$

\begin{tabular}{cc}
\multicolumn{2}{c}{ and $\mathrm{M}=0.65$} \\
\hline Scheme & $\begin{array}{c}\text { Shock Location on } \\
\text { Upper Surface }\end{array}$ \\
\hline TVD FDM & $0.22 \mathrm{c}$ \\
Roe FVM & $0.3 \mathrm{c}$ \\
ANSYS- & No shock wave \\
FLUENT & \\
software & \\
\hline
\end{tabular}

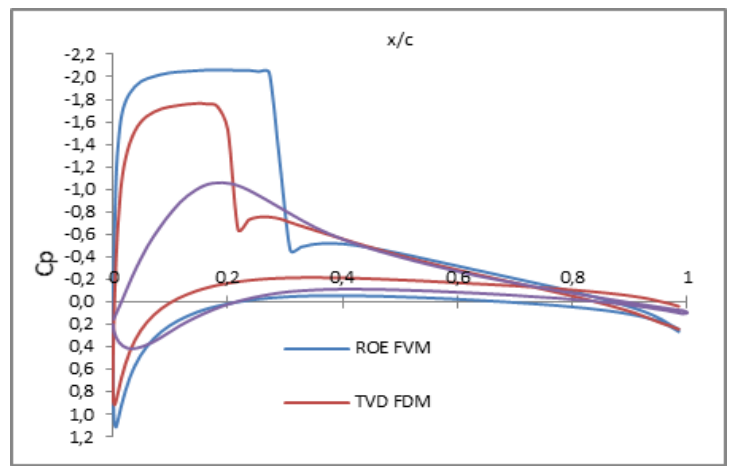

Figure 6. Pressure coefficient distribution at $\alpha=5^{\circ}$ and $M=0.65$ 


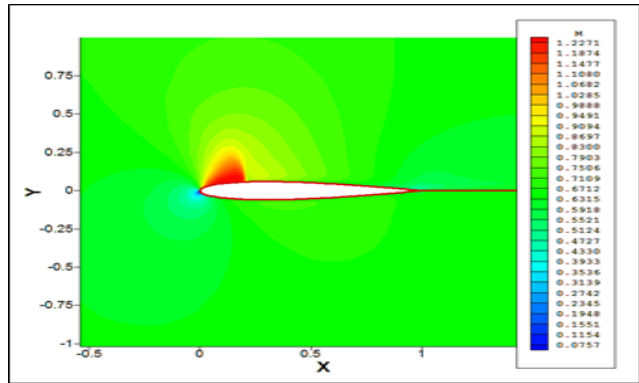

(a)

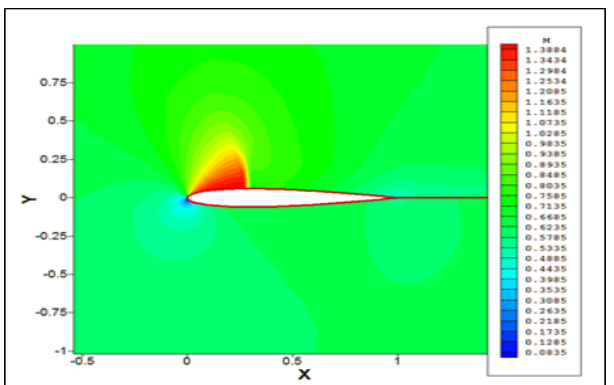

(b)

Figure 7. The Mach number contour near the NACA 0012, at $\alpha=5^{\circ}$ and $M=0.65$ through (a) TVD FDM and (b) Roe FVM

\subsection{Air Flow on NACA 0012 Airfoil for $\mathrm{M}=0.8$ and $\alpha=0^{\circ}$}

When the Mach number was increasing to 0.8 and the angle of attack was kept at $\alpha=0^{\circ}$, three computer codes produce the presence of the shock wave as indicated in Figure 8. The Roe FVM code predicts the position of shock wave nearly at the same location as obtained by ANSYS-FLUENT software. While that, TVD FDM code is slightly further downstream. However, in terms on how the pressure coefficient was distributed along the airfoil surface, the two developed computer codes produced nearly the same result but differ significantly with ANSYS-FLUENT software. This is due to their difference in predicting the shock location as shown in Table 2. In terms of capability in producing sharp pressure gradient at the shock, the developed computer codes are better than the result produced by ANSYS-FLUENT software. In terms of the Mach number, comparison results are shown in Figure 9. These two figures clearly indicate that the TVD FDM code and Roe FVM code give nearly similar flow pattern. However, the shocks produced by TVD FDM code were bigger than Roe FVM code.

Table 2. Comparison result shock position of NACA

\begin{tabular}{ll}
\multicolumn{2}{c}{0012 at $\alpha=0^{\circ}$ and $\mathrm{M}=0.8$} \\
\hline Scheme & Shock location \\
\hline \multirow{2}{*}{ TVD FDM } & Upper surface $0.68 \mathrm{c}$ \\
& Lower surface $0.66 \mathrm{c}$ \\
Roe FVM & Upper surface $0.52 \mathrm{c}$ \\
& Lower surface $0.50 \mathrm{c}$ \\
ANSYSY-FLUENT software & Upper surface $0.51 \mathrm{c}$ \\
& Lower sur ace $0.51 \mathrm{c}$ \\
\hline
\end{tabular}

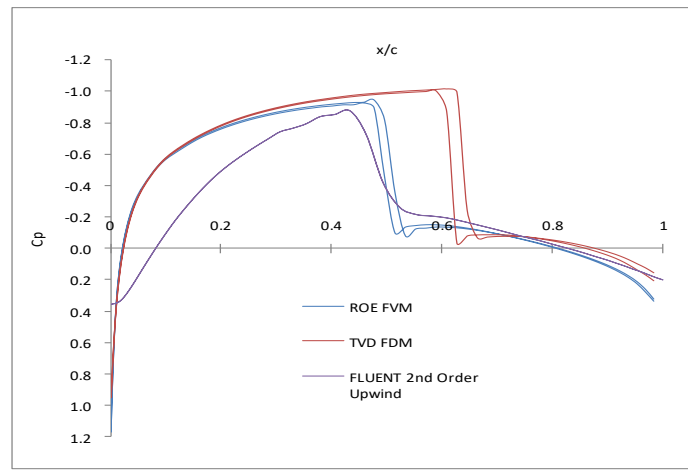

Figure 8. Pressure coefficient distribution for NACA0012, at $\alpha=0^{\circ}$ and $\mathrm{M}=0.8$

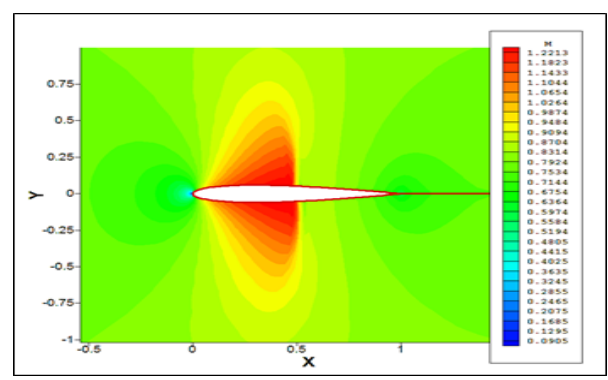

(b)

Figure 9. The Mach number contour near the NACA 0012, at $\alpha=0^{\circ}$ and $M=0.8$ through (a) TVD FDM and (b) Roe FVM

Computational study of flow around a NACA 0012 by using Roe FVM scheme and ... (Fatimah Yusop) 


\subsection{Air flow on NACA 0012 airfoil for $\mathrm{M}=0.8$ and $\alpha=5^{\circ}$}

Different angles of attacks but same Mach number as previously are applied for the case of flow past through NACA 0012. Increasing angle of attack from $\alpha=00$ to become $\alpha=5^{\circ}$, had been found to cause the shock wave to occur at the position further downstream compared to position of shock wave at $\alpha=00$. In terms of pressure coefficient along the airfoil surface, the comparison results between this three software are shown in Figure 10. It is necessary to be noted that the ANSYS-FLUENT software which initially run by use of second order upwind scheme is found to not work. The ANSYS-FLUENT software needs to use a first order upwind scheme. In this respect one can identify that ANSYS-FLUENT software is lacking consistency in their numerical scheme in use

Table 3. Comparison result shock position of NACA

\begin{tabular}{ll}
\multicolumn{2}{c}{0012 at $\alpha=5^{\circ}$ and $\mathrm{M}=0.8$} \\
\hline Scheme & Shock location \\
\hline TVD FDM & Upper surface $0.80 \mathrm{c}$ \\
& Lower surface no shock \\
Roe FVM & Upper surface 0.83 c \\
\multirow{2}{*}{ ANSYS-FLUENT software } & Lower surface no shock \\
& Upper surface 0.63 c \\
& Lower surface no shock \\
\hline
\end{tabular}

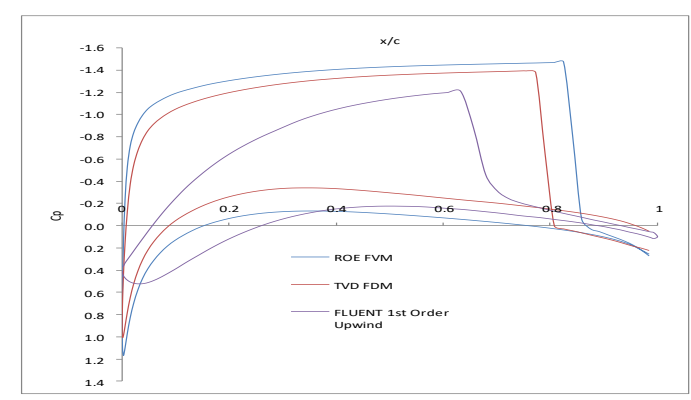

Figure 10. Pressure coefficient distribution, $\mathrm{Cp}$, at $\alpha=$ $5^{\circ}$ and $\mathrm{M}=0.8$

Considering Figure 10 above concludes that the presence of shock wave at the upper surface with their shock location is as given in Table 3. While that, at lower surface, there is no shock wave. The shock location plays an important role, since it will determine the overall pressure coefficient distribution lookalikes. Considering the result of shock location, the two developed computers are able to produce the result which is close to each other but slightly different with the result produced by ANSYS-FLUENT software. Figure 11 show the comparison of Mach number nearly the same results at the given flow condition $\alpha=50$ and $\mathrm{M}=0.8$. These two figures indicate that two developed codes are in good agreement with each other.

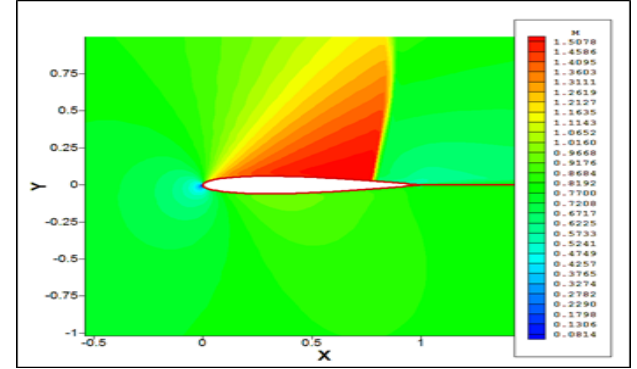

(a)

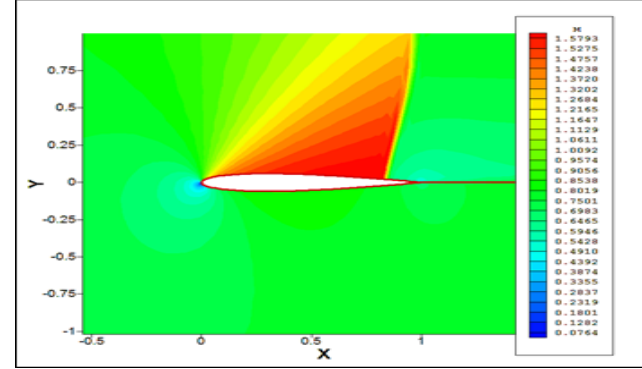

(b)

Figure 11. The Mach number contour near the NACA 0012, at $\alpha=5^{\circ}$ and $M=0.8$ from (a) TVD FDM and (b) Roe FVM

Overall, comparison with ANSYS-FLUENT result indicated the results are quite differing especially to predict the shock. The reason is due the numerical scheme that applied in the software. Each scheme has their own capabilities to capture and predict the discontinuities. High resolution such TVD scheme gives good results but need spend a lot of time.

\section{CONCLUSION}

This paper presents the computational study of flow around a NACA 0012 by using Roe FVM Scheme and Davis-Yee TVD Scheme. Through comparison results to the case of flow past through a 
symmetrical airfoil NACA 0012, it can be concluded that the developed computer code is able to capture the presence of shock wave in the flow field. It seems that the developed computer code much better in capturing discontinuity flow phenomena compare to the ANSYS-FLUENT software by producing a sharp pressure gradient at the shock point. However, comparison with experiment is necessary to ensure that the developed computer code predict correctly the shock location. Since shock position is very important for obtaining a correct value of the overall lift, drag and pitching moment coefficient for the body immersed in the compressible flow field. This represents the suggested for the future work.

\section{ACKNOWLEDGEMENTS}

The author would like to Universiti Tun Hussein Onn Malaysia (UTHM) for the continuous support in this project

\section{REFERENCES}

[1] E. N. Tinoco, "The Impact of High Performance Computing and Computational Fluid Dynamics on Aircraft Development " in 20th Anniversary Dinner \& Symposium CASC Washinton, DC: Coalation for Academic Scientific Computation, 2009.

[2] F. T. Johnson, E. N. Tinoco, and N. J. Yu, "Thirty years of development and application of CFD at Boeing Commercial Airplanes, Seattle," Computers \& Fluids, vol. 34, pp. 1115-1151, 2005.

[3] A. Jameson, W. Schmidt, and E. Turkel, "Numerical solutions of the Euler equations by finite volume methods using Runge-Kutta time-stepping schemes," AIAA Paper, vol. 1259, p. 1981, 1981.

[4] M. Drela, "Newton solution of coupled viscous/inviscid multi element airfoil flows," in 21st Fluid Dynamics, Plasma Dynamics and Lasers Conference: American Institute of Aeronautics and Astronautics, 1990.

[5] H. Ghuanghui, "Numerical simulations of the steady Euler equation on unstructured grid." vol. Ph.D: Hong Hong Baptist University, 2009, p. 148.

[6] M. H. Chen, C. C. Hsu, and W. Shyy, "Assessment of TVD schemes for inviscid and turbulent flow computation," International Journal for Numerical Methods in Fluids, vol. 12, pp. 161-177, 1991.

[7] K. R. Qureshi and C. H. Lee, "Behavior of TVD Limiters on the Solution of Non-Linear Hyperbolic Equation," Modern Physics Letters B, vol. 19, pp. 1507-1510, 2005.

[8] R. AbgrallR, "An extension of Roe's upwind scheme to algebraic equilibrium real gas models". Computers \& Fluids, 19(2), 171-182, 1991.

[9] P. Glaister, "A weak formulation of Roe's scheme for two-dimensional, unsteady, compressible flows and steady, supersonic flows," Computers \& Mathematics with Applications, vol. 30, pp. 85-93, 1995.

[10] C. Hirsh, Numerical Computation of Internal and External Flows (2nd Ed.). Great Britian: Elsevier, 2007.

[11] K. A. Hoffman \& S. T. Chiang, Computational Fluid Dynamics (4th ed. Vol. II). USA: www.EESbooks.com. 2000.

[12] J. C. Tannehill, D. A. Anderson, and R. H. Pletcher, Computational Fluid Mechanics and Heat Transfer, 2nd ed. United State of America: Taylor \& Francis, 1997.

[13] J. Blazek, Computational Fluid Dynamics: Principles and Applications: Elsevier, 2005

[14] A. Harten, "High resolution schemes for hyperbolic conservation laws," Journal of Computational Physics, vol. 49, pp. 357-393, 1983.

[15] T. J. Chung, Computational fluid dynamics: Cambridge university press, 2010.

[16] Y. L. Chang, "Development of a CFD code using TVD schemes and advanced turbulence models for incompressible flow simulations." Ph.D: Michigan Technological University, 1997, p. 107.

[17] S. F. Davis, "A Simplified TVD Finite Difference Scheme via Artificial Viscosity". SIAM Journal on Scientific and Statistical Computing, 8(1), 1-18, 1987.

[18] H. C. Yee and A. Harten, "Implicit TVD schemes for hyperbolic conservation laws in curvilinear coordinates," AIAA Journal, vol. 25, pp. 266-274, 2015/07/29 1985.

[19] S. Chakravarthy and S. Osher, "A new class of high accuracy TVD schemes for hyperbolic conservation laws," in 23rd Aerospace Sciences Meeting: American Institute of Aeronautics and Astronautics, 1985.

[20] K. Anderson, J. L. Thomas, and B. Van Leer, "Comparison of finite volume flux vector splitting for the Euler equations," AIAA Journal, vol. 24, pp. 1453-1460, 1986.

[21] A. Jameson, "Requirements and trends of computational fluid dynamics as a tool for aircraft design." Proceedings of the 12th NAL symposium on aircraft computational aerodynamics. Tokyo, Japan. 1994.

[22] H. Shigeki, A. Justin, \& H. Klaus, "Development of a modified Runge-Kutta scheme with TVD limiters for the ideal two-dimensional MHD equations". 36th AIAA Aerospace Sciences Meeting and Exhibit. American Institute of Aeronautics and Astronautics. 1998.

[23] P. Glaister, "Flux difference splitting for the Euler equations in one spatial co-ordinate with area variation." International Journal for Numerical Methods in Fluids. 1988. 8: 97-119.

[24] S. I. Sohn, A new TVD-MUSCL scheme for hyperbolic conservation laws. Computers \& Mathematics with Applications, 50(2), 231-248, 2005.

[25] L. Mottura, L. Vigevano, \& M. Zaccanti, “An Evaluation of Roe's Scheme Generalizations for Equilibrium Real Gas Flows”. Journal of Computational Physics, 138(2), 354-399M 1991. 